\title{
la energía nuclear y la industria en Francia
}

\section{A. Roux}

$591-6$

\section{sinopsis}

En este artículo se analizan los efectos favorables que para la industria tiene el desarrollo de la energía nuclear y las interacciones entre ambas.

Se hace referencia al problema del aprovisionamiento energético en Francia y a la necesidad de un esfuerzo en materia de ahorro de energía, al desarrollo de nuevas fuentes y a la prioridad en el establecimiento de un importante programa de centrales nucleares.

También es analizado el papel de la Industria, la Comisaría de Energía Atómica y Electricidad de Francia en el abastecimiento y en la transformación de los materiales nucleares.

Por último, se hace un repaso general de los medios humanos y técenicos con que se cuenta on la actualidad, asi como de las inversiones previstas para un desarrollo nacional de esta energía.

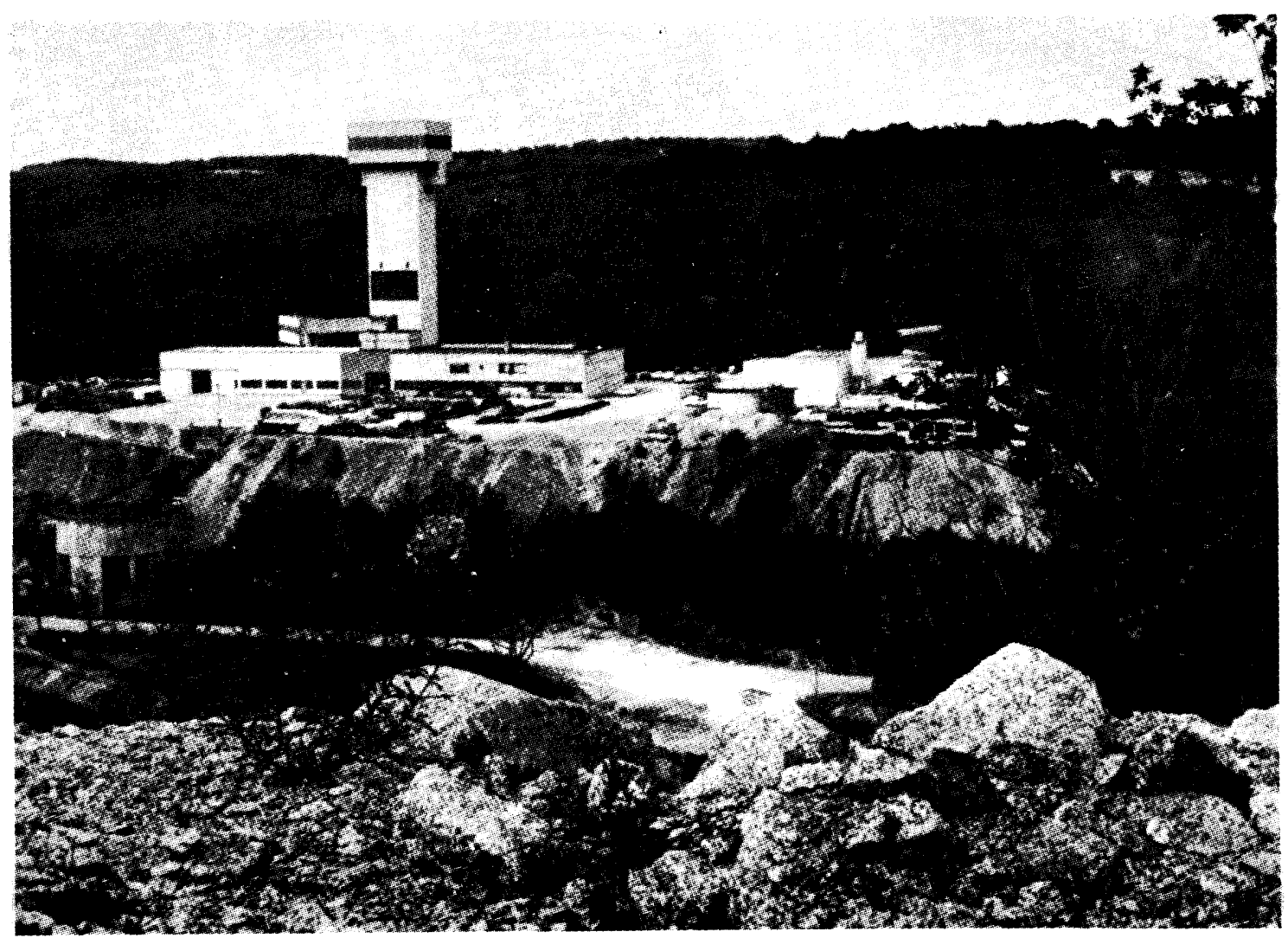

Mina de uranio de la Crouzille; pozo de la Margnac. 


\section{INTRODUCCION}

Podríamos definir la industria como el conjunto de medios de producción de bienes y de servicios que exigen un mínimo de organización y concentración:

Se sirve de los hombres, de las máquinas, de las materias primas y de la energía.

Su historia se confunde con la de las técnicas, pues no existe hasta ahora un ejemplo de un descubrimiento útil que no se haya beneficiado rápidamente, para su puesta en el mercado, de una tecnologia apropiada y de los medios de financiación adecuados.

El pasado nos proporciona numerosos ejemplos de técnicas que produjeron grandes transformaciones en las condiciones de vida del hombre y en su organización socio-política, pero que en un principio tropezaron con el escepticismo y con una cierta hostilidad de quienes más tarde serían sus beneficiarios, y que aportaron junto con inestimables beneficios una secuela de molestias, de peligros e, incluso, de muerte.

Ninguna de estas técnicas levantó, sin embargo, tantas polémicas como la energía nuclear; en efecto, a los incendios, a las explosiones de calderas no se les ha dado nunca tanta importancia, mientras que el átomo produce miedo y polariza las reacciones de rechazo de los que se inquietan por el futuro de nuestra civilización.

\section{EL UNICO MEDIO A NUESTRO ALCANCE PARA HACER FRENTE A LA CRISIS DE LA ENERGIA}

Este reflejo de rechazo es perjudicial para un juicio objetivo sobre un sector tan importante, que es uno de los pocos en los que Francia dispone de una apreciable tecnología avanzada, y que constituye, sin lugar a dudas, el único medio a nuestro alcance para llegar, sin retroceder de una manera dramática para todos, al tiempo todavia lejano en que el agua y el sol permitan de nuevo la abundancia.

El aprovisionamiento de energía en Francia, en los próximos veinte años supone, en efecto, un grave problema.

La energia primaria consumida por Europa Occidental alcanzó la cifra de 1,03 miles de millones de toneladas equivalentes de petróleo en 1973. Después de haber conocido desde 1965 un ritmo de crecimiento de un $5 \%$ al año, una reducción de este ritmo, a un $3 \%$ al año, nos llevará a que en el año 2000 habrá un consumo de 2,73 miles de millones de toneladas equivalentes de petróleo.

Suponiendo que las producciones autónomas de carbón, de gas natural y de petróleo fuesen impulsadas al máximo, que el ritmo de construcción de centrales nucleares fuese de $20.000 \mathrm{MW}$ al año, que se adquiriese carbón en el exterior por un valor de cien millones de toneladas equivalentes de petróleo y que las importaciones de gas aumentasen de forma considerable, el déficit petrolífero de Europa Occidental pasaria a un mínimo anual de 550 millones de toneladas en 1985 , pero superaría los 10,85 miles de millones de toneladas en el año 2000. Esta cifra supondría el $60 \%$ de la energía petrolífera disponible en el mercado internacional en una época en que las necesidades del tercer mundo y de los paises productores se habrán incrementado sensiblemente.

No es necesario recordar que Francia, ante esta perspectiva tan sombria, se encuentra en una situación aún más precaria, pues no dispone ni del carbón del Ruhr, ni del petróleo del Mar del Norte. 


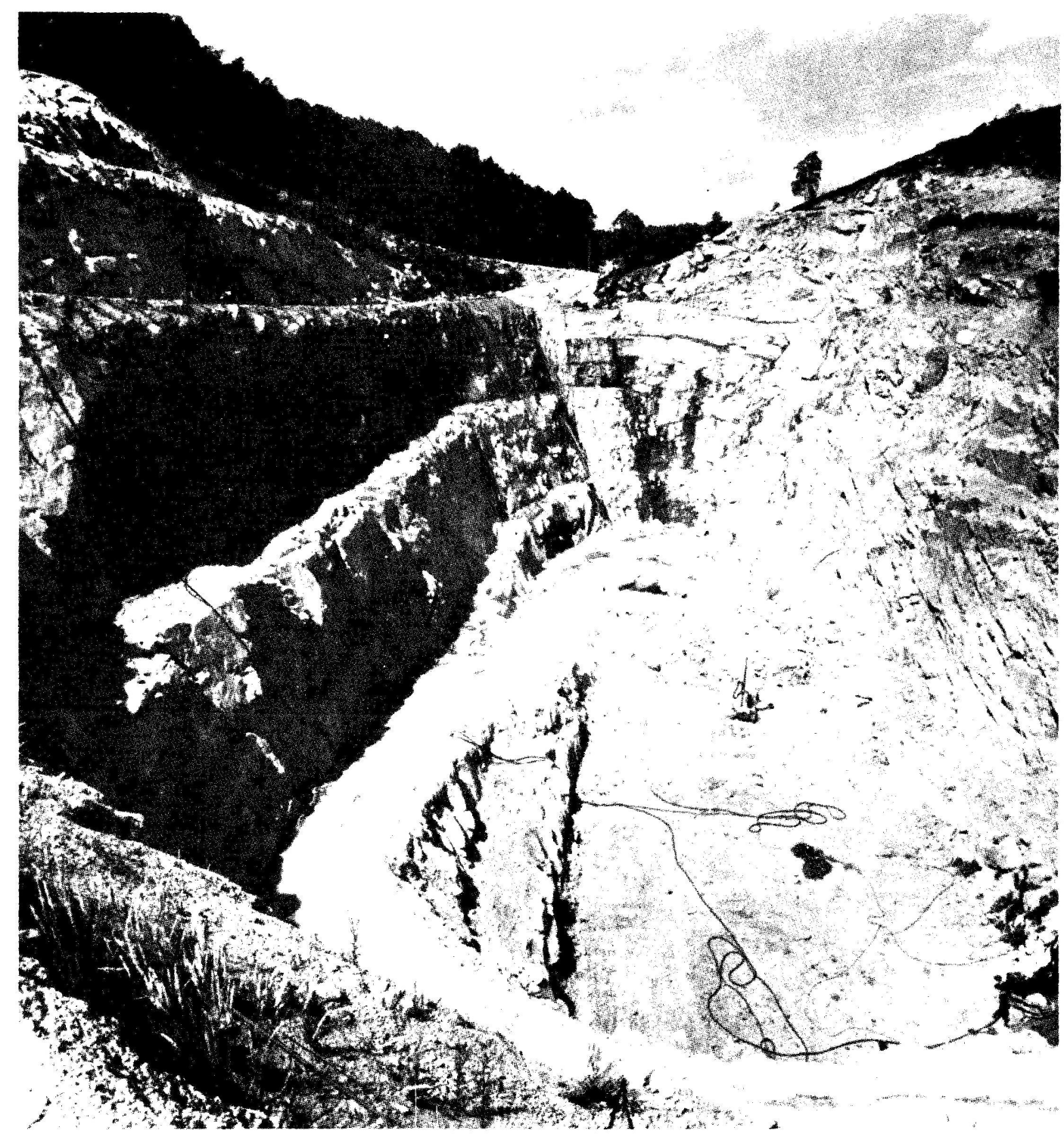

Cantera de Peny, explotación a cielo abierto.

Si se quiere evitar la caída en picado de la industria francesa, conservando un mínimo de independencia energética, se impone realizar un gran esfuerzo. Primero, habrá que economizar energía en todos los campos: Los cambios actuales encaminados hacia vehículos de menor consumo, hacia viviendas con un mejor aislamiento, hacia procedimientos de construcción menos costosos en cuanto a energía, hacia una sustitución por productos más económicos en el terreno de la fabricación, deberán proseguirse a toda costa.

Francia tendrá que dedicar una parte importante de sus medios de investigación y de sus inversiones al desarrollo de nuevas energías y a la recuperación de aquéllas que ahora se desaprovechan: la geotérmica, las mareas, la solar, la utilización de las fuentes termales y todo lo que el ingenio del hombre pueda sugerir. Sin embargo, estas economías y estas nuevas energías no serán sino pequeños remedios. En efecto, las dimensiones del problema son grandes: la superficie que habrá que sacrificar para producir $1.000 \mathrm{MW}$ mediante energía solar se cifra en miles de hectáreas; las zonas geotérmicas que se puedan explotar son muy difíciles de encontrar y muy caras de equipar; la energía hidráulica ya ha dado en Francia todo lo que podía dar; la combustión de los desechos es pobre en calorias y muy contaminante. 
A menos que se redujese, por medio de restricciones draconianas, el consumo de energía de los particulares en detrimento de su nivel de vida, y el de la industria, al precio de una baja competitividad que agravaria la crisis de empleo, se impone un importante programa de construcción de centrales nucleares en los próximos años.

\section{UNA VOLUNTAD DE ENFRENTARSE AL PROBLEMA}

Este programa depende sobre todo del deseo del Gobierno y de la Comisaria de Energía Atómica que deben simultáneamente abrir paso a los indispensables recursos de financiación, elaborar las técnicas necesarias para que el programa se pueda llevar a cabo, garantizar la reserva de uranio, proveerse de todo lo necesario para los ciclos de combustible y organizar un control capaz de dar una respuesta a los que se preguntan acerca de los riesgos que esta energía comporta en la actualidad y en el futuro.

Depende igualmente del organismo Electricidad de Francia que es el encargado de construir las centrales nucleares, de su explotación y de la distribución de la energía producida.

Depende finalmente del deseo de la industria de enfrentarse a este problema.

Después de un retraso debido a la guerra, durante el cual las industrias de los países anglosajones fueron prácticamente los únicos en iniciar la era nuclear, la industria francesa realizó sus primeras armas hacia mediados de los años 50 con la construcción de los reactores de Marcoule.

En efecto, es durante esta época cuando se abrieron las Escuelas de Ingenieria Nuclear de la C.E.A. y se ofreció a los primeros equipos privados la responsabilidad de sectores importantes en el reactor y en las instalaciones de producción de energía.

A partir de entonces, la competencia y las responsabilidades de la industria han ido creciendo hasta alcanzar el estado de madurez actual que le autoriza a competir en el mercado mundial con cualquier tecnología avanzada.

\section{LA RESPUESTA DE LA INDUSTRIA AL DESAFIO NUCLEAR}

La industria francesa está presente en el origen mismo del ciclo, a nivel de prospección y de explotación minera.

El aprovisionamiento de Francia en material nuclear es una de las misiones de la C.E.A., aunque también tiene ayuda de algunas firmas privadas asociadas.

\section{LA MINA}

Grandes grupos mineros desempeñan un importante papel en la prospección de los minerales de uranio en Francia y en los paises extranjeros con los que ha podido establecerse una colaboración. En Francia, la parte de recursos descubiertos por sociedades privadas sobrepasa el $15 \%$ y representa 2,5 veces el consumo anual del país en 1980.

La participación de la industria en la actividad minera, propiamente dicha, es relativamente escasa, pues en 1975 no llegaba en Francia a más de un $10 \%$. Sin embargo, las filiales de los grandes grupos químicos intervienen de manera más amplia en la fase de elaboración de concentrados, bien en Francia, o bien en las explotaciones mineras extranjeras en las que Francia tiene intereses. 


\section{EL COMBUSTIBLE}

Como hemos visto, el papel de la industria es bastante modesto adquiriendo una importancia mucho mayor en la fase de elaboración propiamente dicha del combustible que comprende el refino del uranio, su acondicionamiento, su envainado y, si es preciso, su enriquecimiento.

Los datos técnicos que sirven para realizar los procedimientos básicos proceden de la C.E.A. o de algún asociado con licencia extranjera, pero es en esta fase del combustible, antes de su utilización, cuando el talento artístico de los industriales se manifiesta.

La calidad de los elementos combustibles es primordial para la fiabilidad y seguridad de funcionamiento de los reactores productores de energía, así como de los reactores de investigación; es en su fabricación donde aparecen por primera vez, en el curso del proceso total, el rigor y el cuidado especiales que caracterizan la técnica nuclear.

Dejando aparte los elementos de plutonio, fabricados directamente por la C.E.A., los elementos combustibles de todos los reactores electrógenos construidos en Francia provienen hoy en día de una industria cuyas instalaciones evolucionan continuamente, a partir de los talleres con carácter seudo-artesanal de donde salieron hace veinte años los elementos de aletas de los primeros reactores de uranio natural refrigerados por gas.

Hoy día, los elementos combustibles con potencias caloríficas elevadas para el programa nuclear francés se fabrican industrialmente en condiciones normales de precio y calidad por las filiales de grandes grupos químicos o metalúrgicos franceses.

Debemos hacer una mención especial del enriquecimiento del uranio: los tipos de reactores usados actualmente en Francia para la producción de electricidad consumen un uranio más o menos rico en isótopo 235.

Dejando aparte las instalaciones de enriquecimiento de pequeña $\circ$ mediana capacidad, construidas para satisfacer las necesidades militares, el aprovisionamiento de óxido de uranio enriquecido necesario para las centrales electrógenas es actualmente un monopolio de los Estados Unidos provistos de secretos técnicos hasta ahora celosamente guardados.

Un legítimo deseo de independencia económica ha llevado a Francia a organizar su propio proceso de enriquecimiento y a decidir la construcción de fábricas capaces de cubrir sus necesidades a la vez que las de algunos paises asociados.

Aunque el método de enriquecimiento fue elaborado brillantemente por la C.E.A., no hubiera podido llevarse a cabo sin la colaboración de las distintas especialidades industriales; la exigencia en materia de potencia, de fiabilidad y de precio sobrepasan todo lo que se había pedido hasta ahora; los problemas a resolver parecian gigantescos y las dificultades insuperables. 
Gracias a la experiencia sacada de la construcción de Pierrelatte los industriales franceses pudieron, sin embargo, responder a las demandas de Eurodif para la construcción de la fábrica de Tricastin, a pesar de la magnitud de los problemas técnicos planteados en campos tan distintos como: la calderería, los compresores, las juntas de impermeabilidad y muy en especial la cerámica. Utilizada para las barreras de difusión a un nivel de calidad muy superior al acostumbrado en esta industria, la cerámica constituia, desde luego, un elemento clave de este conjunto que necesitaba la fabricación a gran escala de tubos porosos cuidadosamente estudiados durante años por los laboratorios públicos y los mejores equipos privados.

\section{LAS CENTRALES}

La mayor participación industrial en el sector nuclear se produce evidentemente en el campo de las centrales eléctricas en las que a las dificultades debidas al peso y a las dimensiones se suman los grandes controles siempre presentes en este terreno.

Es en el campo de la construcción de las centrales nucleares donde mejor puede medirse la evolución sufrida por la industria francesa, que pasa del papel de simple proveedor de componentes al de estar capacitado actualmente para exportar tecnologia.

La distribución del coste de un grupo de $1.000 \mathrm{MW}$ es, aproximadamente, de un $40 \%$ para la parte nuclear, de un $40 \%$ para la parte convencional y de un $20 \%$ para la ingeniería civil. Solamente la parte de ingeniería civil de la obra representa alrededor de 20 millones de horas de trabajo.

Después de algunas dudas, Francia decidió establecer su programa nuclear para los próximos diez años únicamente sobre la técnica de agua a presión prosiguiendo la puesta a punto de los supergeneradores de neutrones rápidos con el fin de permitir una utilización correcta de las reservas de uranio.

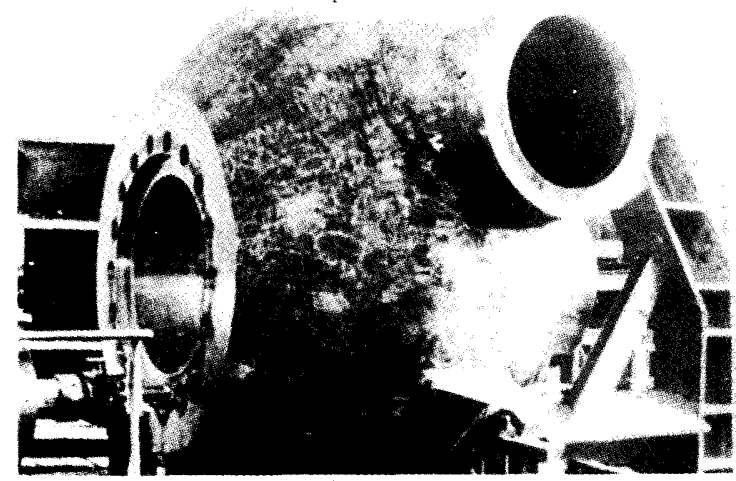

Bomba primaria

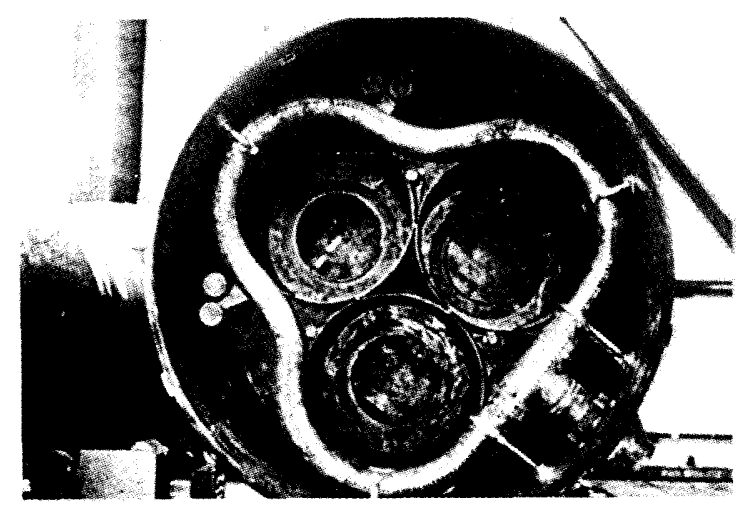

Generador de vapor con el montaje interior

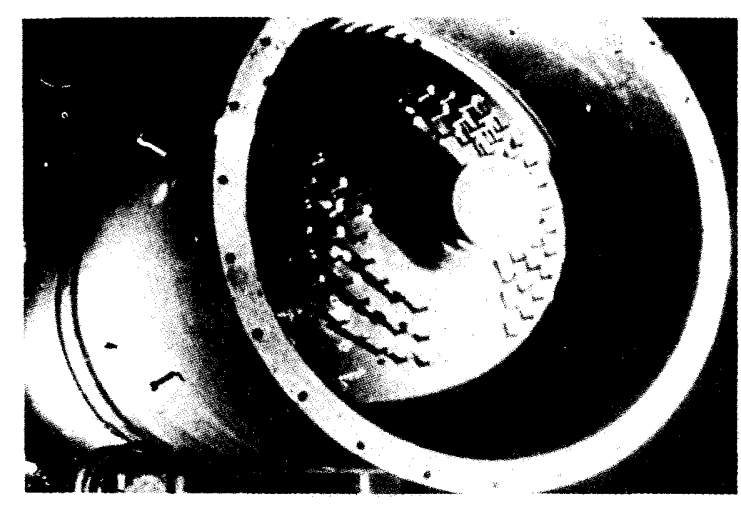

Presurizador para Fesselheim.

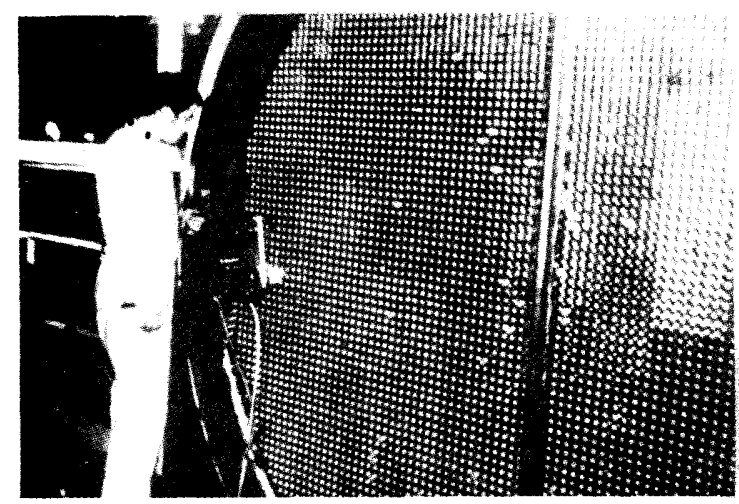

Generador de vapor. Soldadura primera pasada. 


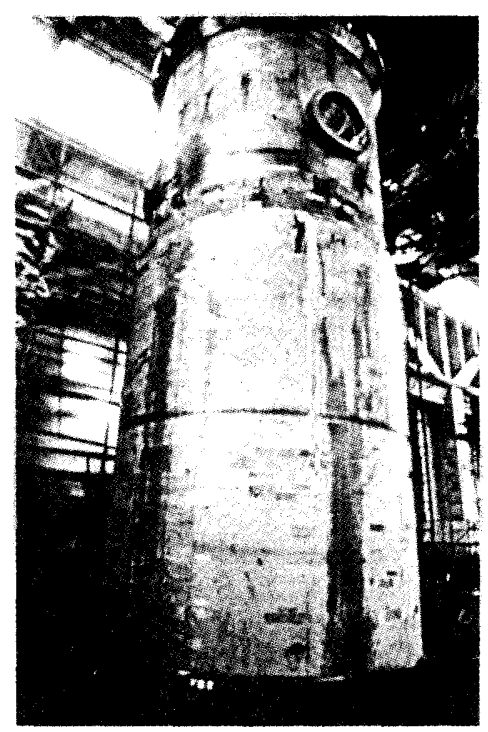

Envoltura del núcleo central.
En estas dos familias de reactores, la parte nuclear es, ante todo, calderería.

La vasija de un reactor de $900 \mathrm{MW}$ pesa $330 \mathrm{t}$, tiene $12 \mathrm{~m}$ de alto y $4 \mathrm{~m}$ de diámetro.

Su fabricación, su transporte y su montaje habrian podido producir un estrangulamiento importante de la actividad si la industria francesa no hubiese hecho los esfuerzos necesarios para ser capaz de realizar este tipo de grandes piezas que forman probablemente los más importantes conjuntos transportables fabricados hasta ahora por el hombre en el campo del equipamiento industrial.

La fabricación de las vasijas de acero de los grandes reactores está hoy dia totalmente superada en Francia a la espera de que la evolución dimensional imponga los cajones de hormigón construidos in situ, para lo cual las empresas francesas de ingeniería civil están técnicamente preparadas.

Los generadores de vapor, con sus $310 \mathrm{t}$ y sus $20 \mathrm{~m}$ de altura no le van a la zaga a la vasija en el plano dimensional.

Estos tienen grandes problemas de impermeabilidad a nivel de las placas tubulares que hicieron sufrir a toda una generación de ingenieros, pero que hoy día están igualmente resueltos.

Desde que Edison hizo, a finales del siglo pasado, la primera distribución pública de energía eléctrica, los fabricantes de turboalternadores tuvieron que ir perfeccionando periódicamente su técnica con el fin de aumentar la potencia unitaria de sus máquinas, ya que sus necesidades se duplicaban cada diez años.

La aparición de la energía nuclear trastornó esta ley que se había convertido en rutinaria; los grupos de $125 \mathrm{MW}$ de Porcheville desaparecieron hace veinte años; los pedidos actuales son de $900 \mathrm{MW}$ o incluso de $1.300 \mathrm{MW}$.

Este problema dimensional planteado a los fabricantes de turbinas se agravó más aún por la calidad más bien pobre del vapor que arrastra, con la misma potencia, caudales dos veces más importantes y que crean problemas de condensación en caso de fugas.

Las cifras son quizás más espectaculares que en caldereria, ya que el peso de las piezas más grandes es del orden de $200 \mathrm{t}$, pero se trata de turbinas o de rotores que giran a 1.500 vueltas por minuto con diámetros de los álabes de cerca de 10 metros.

Como los fabricantes de vasijas, los fabricantes de turbinas poseen la técnica y las instalaciones necesarias para hacer frente a la demanda del mercado nacional e incluso poder exportar.

A título indicativo, la capacidad de producción del principal constructor francés de turboalternadores le sitúa entre los mejores del mundo.

Sin embargo, la construcción de las centrales nucleares no afecta exclusivamente a los fabricantes de vasijas y de turbinas. Otros industriales y especialistas participan con éxito en estos vastos complejos que necesitan, por ejemplo, de la perfección de la calderería de acero inoxidable -especialmente para la vasija-, los circuitos y los intercambiadores de los supergeneradores, de la técnica de refrigeración por metales líquidos que ocasiona problemas técnicos poco corrientes, de la mecánica de precisión para la manipulación de 
combustibles activos, 0 del telecontrol y de la informática, primordiales en materia de protección ya que las señales de alarma dependen de ellos.

La cadena de producción de la energía nuclear no se detiene en el transformador de salida y la piscina de desactivación de la central.

Por el contrario es en esta fase donde aparecen por primera vez esos famosos productos de fisión radiactivos generadores de riesgos potenciales que mantienen la controversia sobre la oportunidad de grandes programas nucleares.

Dentro del reactor, el uranio se carga de productos de fisión contra los cuales hay que protegerse. Hay que transportarlo, tratarlo y después resolver el problema de la evacuación o del almacenamiento de los últimos residuos.

Ese sector del ciclo del combustible nuclear, bajo la responsabilidad de la C.E.A., ha ido progresando gracias a una participación activa de la industria.

Esta participación se manifiesta, por ejemplo, en el tratamiento del combustible irradiado garantizado por COGEMA, en la realización, a cargo de expertos, de las fábricas de tratamiento como la del Hague o en el campo del transporte.

Si añadimos a la lista antes citada los laboratorios de alta actividad y el empleo de radio-isótopos no queda prácticamente nada en el sector industrial que no sea afectado por la técnica nuclear como proveedor, como prestador de servicios o como usuario.

Los sectores más importantes de esta lista son indudablemente la calderería, la mecánica, la electrotécnica, la electrónica y la química, pero existen otros entre los que no hay que olvidar la ingeniería civil que ha tenido que poner a punto el hormigón pesado para la protección antirradiaciones y concebir recintos de contención de gran volumen, la industria de los plásticos y de los elastómeros presente en todos los problemas de impermeabilidad y en los grandes refrigerantes atmosféricos, e incluso la industria textil para ciertas prendas de trabajo en ambiente radiactivo.

Aparte de las industrias que utilizan los rayos gamma, la industria no es, en general, cliente directo de lo nuclear. Interviene como proveedor de bienes y de servicios, aporta productos, investigaciones e ingenieria.

Estos dos últimos aspectos han alcanzado en Francia un desarrollo más tardío que en Estados Unidos o Alemania. En Francia es a la C.E.A. a la que corresponde la misión de investigar y poner a punto los procedimientos nucleares. Mientras que Electricidad de Francia se encarga del diseño industrial de las centrales que construye para sus propias necesidades.

Este método de trabajo, que da la pauta a seguir en grandes proyectos públicos, se comenzó a usar en los años 50 . Ha tenido muchos aspectos positivos, ya que la industria francesa no estaba en condiciones de gastar como las industrias alemanas o americanas las enormes sumas necesarias para la adquisición de las técnicas nucleares, ni de reunir en los programas, en principio reducidos, a los numerosos equipos de ingenieria necesarios para la realización de las principales unidades productoras de energía.

Tan conscientes como las propias industrias, de las imperiosas necesidades de exportación, la C.E.A. y la E.D.F. aceptaron, sin embargo, rápidamente la asociación de la industria a sus trabajos confiándole por ejemplo el diseño industrial del reactor y la realización, bajo su responsabilidad, de elementos cada vez más importantes del interior de una central.

Esta política permitió a la industria crear sus propias oficinas de proyectos que actualmente pueden equipararse con sus homólogas extranjeras sin miedo a exagerar.

La presencia de la C.E.A. o de la E.D.F. en los organismos encargados de la exportación tranquiliza a los eventuales clientes en el plano técnico. 
- səjeuolo!r zir әfuejsqo ou odəd

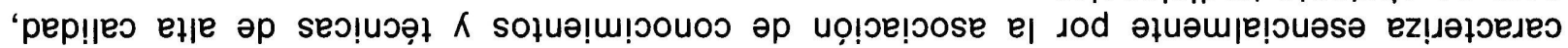

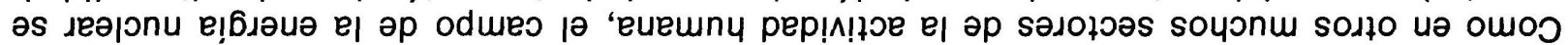

‘

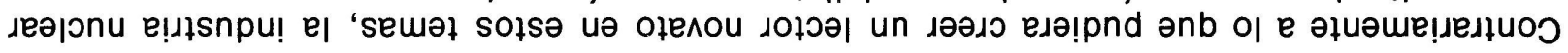

\section{SэygwOH SO7}

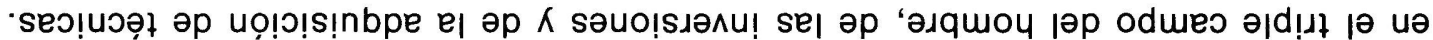

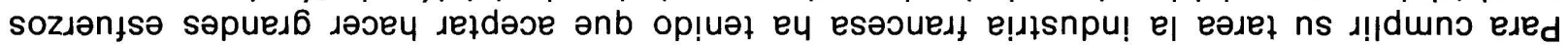

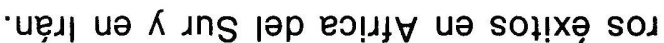

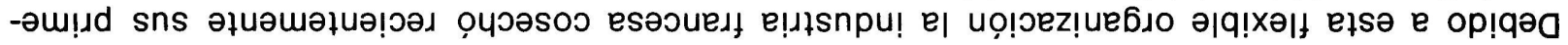

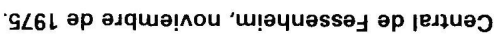

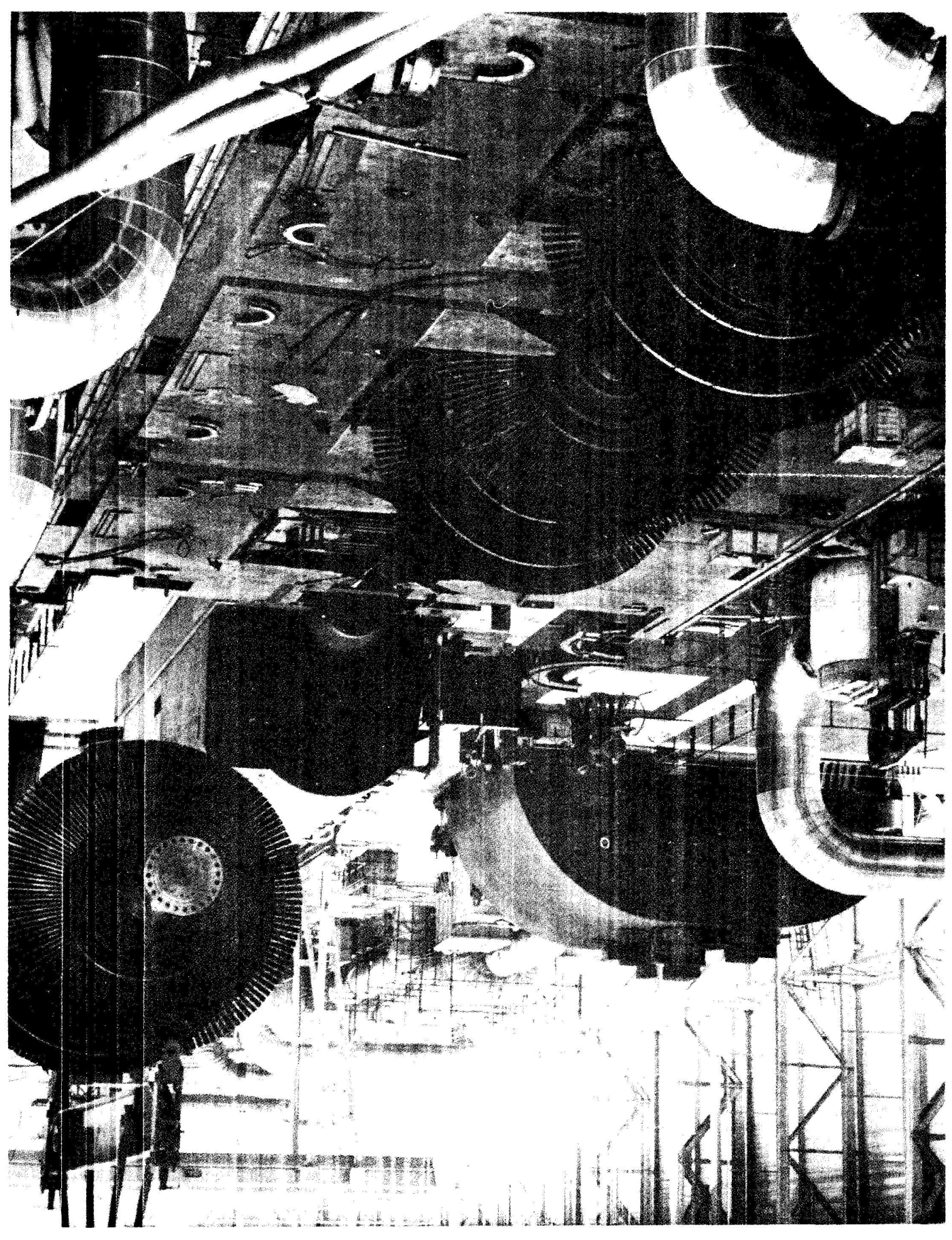


Las grandes vasijas de los reactores tienen, por ejemplo, problemas de resistencia de materiales y de resistencia a la corrosión. Es necesario laminar chapas de gran espesor mediante soldaduras homogéneas, eliminar las tensiones internas, determinar los puntos frágiles para consolidarlos, etc. La degradación de las características del acero bajo los efectos de las radiaciones deberá tenerse en cuenta, aunque no constituye el problema más importante.

El enriquecimiento del uranio nos lleva a problemas de mecánica de fluidos, de corrosión química, de tratamiento especial de la cerámica.

Los problemas en las fábricas de tratamiento son problemas ordinarios de ingeniería quími$\mathrm{ca}$, tratando substancias peligrosas con las que se emplean precauciones para que no sobrepasen sus puntos críticos.

A priori, los ingenieros y los responsables de las industrias que trabajan para lo nuclear podrian permitirse el lujo de desconocer totalmente las características particulares de este sector.

En la práctica es muy diferente, pues en muchos casos tanto el que concibe como el que ejecuta deben, para ser eficaces, tener la cultura nuclear adecuada.

No es necesario, pero sí aconsejable, que tanto los ingenieros como los industriales estudien durante cierto tiempo lo nuclear para que algunos conceptos lleguen a ser intuitivos.

La impureza que degrada el balance neutrónico, puesto que puede activarse peligrosamente, debe llamar la atención así como la enorme tensión térmica debida a la rotura de un circuito de refrigeración o bien la fuga que puede contaminar un recinto de contención y que luego necesita una descontaminación bastante costosa.

Esta cultura que origina reacciones intuitivas marca la diferencia entre una industria cuyos ingenieros estén "nuclearizados" y otra en que no lo estén.

Hoy Francia puede estar orgullosa de ser uno de los pocos países del mundo que dispone de un importante cuerpo de ingenieros especializados en energía nuclear, tanto en la propia industria como en la C.E.A. y en E.D.F.

Esta situación es naturalmente el resultado de la experiencia adquirida después de veinticinco años trabajando en uno de los programas nucleares más importantes del mundo.

Se debe también al esfuerzo de formación seguido por la C.E.A. en sus principales centros $y$, especialmente, en el I.N.S.T.N. de Saclay.

Desde la primera promoción salida en 1955, el I.N.S.T.N. no ha cesado de extender entre los ingenieros franceses esa cultura nuclear que hace que sea posible pensar en la exportación.

A este esfuerzo de formación pura se une, naturalmente, la experiencia adquirida en las oficinas de proyectos de la industria a las que se han confiado con-

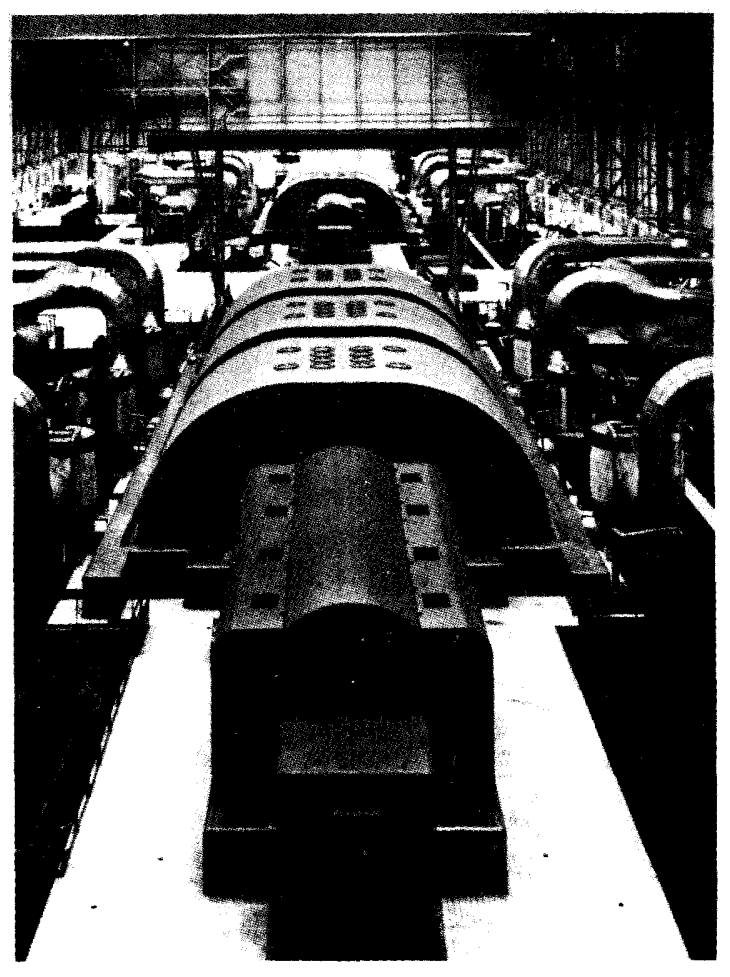

Sala de máquinas de la central de Fessenheim. 
tratos de investigación y la que se adquiere mediante los múltiples contactos entre ingenieros de los distintos grupos franceses y sus colegas americanos.

Bien situada en cuanto a hombres con talla de grandes sabios y de técnicos de alto nivel gracias a la C.E.A. y a las Universidades, Francia también lo está en cuanto a la industria. La fuerza de un ejército reside, sin duda, en el talento de los generales, pero también depende de la competencia de los capitanes y de la experiencia de los ayudantes.

Desde este punto de vista, después de veinticinco años de esfuerzos, la industria francesa se encuentra en muy buena posición.

\section{INVERSIONES CONSIDERABLES}

Para poder producir y vender una industria necesita tanto hombres como medios.

En el plano de las inversiones, los esfuerzos de la industria francesa han sido bastante considerables y costosos.

Como para los hombres, estas inversiones se han realizado sobre todo en los medios clásicos, pues estos medios clásicos constituyen hoy en día la condición necesaria y suficiente para hacer energía nuclear a nivel mundial.

Hace quince años, la industria francesa no disponía de talleres provistos de aparatos de elevación, medios de soldadura y medios de control suficientes para construir las grandes vasijas de reactores: las fábricas Creusot están capacitadas en la actualidad para fabricar ocho vasijas al año y una veintena de generadores de vapor.

Hace quince años los constructores de turboalternadores estaban dispersos. Hoy, Alsthom Atlántica puede producir grupos de $1.300 \mathrm{MW}$ eléctricos a un ritmo anual de $10.000 \mathrm{MW}$.

En otros campos la industria se ha equipado con éxito para poder responder a las necesidades de la separación de isótopos; se han construido nuevas fábricas para la producción de barreras de difusión al astronómico ritmo exigido por la importancia de los programas de Eurodif.

El conjunto de estos programas de inversiones constituye, sin lugar a dudas, un desafío de cara al porvenir; pesa bastante sobre el balance global de la colectividad nacional, pero sin embargo ahora existe el instrumental y la industria francesa puede utilizarlo pensando fuera de sus fronteras.

\section{UN GRAN ESFUERZO EN LA ADQUISICION DE TECNICAS}

El último esfuerzo, aunque no por eso menor, ha sido el realizado por la industria para adquirir las técnicas necesarias en este campo. Este esfuerzo lo ha hecho en sus propios laboratorios, en sus oficinas de proyectos, mediante licencias extranjeras o enviando a sus ingenieros para estancias de larga duración allí donde pudieran aprender.

Estas técnicas, unas veces clásicas, otras especificas, han traido progresos que desbordan el sector.

Gracias a la energia nuclear, la industria francesa ha hecho importantes progresos en muy diferentes campos:

- Domina en este momento las técnicas más modernas en calderería pesada con un alto grado de seguridad.

- Sabe actualmente manejar materiales de gran pureza y materiales altamente tóxicos.

- Se ha habituado a trabajar industrialmente en condiciones de una fiabilidad excepcional que superan las de la aeronáutica o las de la electrónica.

- Ha hecho espectaculares progresos en el campo de la cerámica y en el de la ingeniería química. 
- Por último, los problemas de radiactividad, de protección contra radiaciones ionizantes y de tratamiento de residuos activos han dejado de ser gracias a estos progresos esotéricos.

El conjunto de estos conocimientos, raras veces patentables, constituye un "saber hacer" que sitúa a la industria francesa a la altura de las más desarrolladas del mundo.

\section{CONCLUSION}

¿Se podría concluir brevemente un tema con un horizonte tan vasto?

En primer lugar, la industria francesa está actualmente capacitada para afrontar con éxito el indispensable programa de construcción de centrales nucleares que Francia debe acometer urgentemente para asegurar sus necesidades energéticas, sin sacrificar su independencia o su nivel de vida.

Después de veinticinco años de esfuerzos técnicos, humanos, financieros, las empresas francesas de metalurgia, mecánica, electrotécnica, electrónica, química e ingeniería civil disponen de hombres, medios y de la categoria necesaria para hacer frente a las demandas de los grandes clientes nacionales.

En segundo lugar, la industria francesa es hoy capaz de competir dentro del sector nuclear con las mejores industrias extranjeras. Es fiable, tiene referencias y dispone de los hombres y medios necesarios. Mantendrá esta buena posición mientras el mercado interior le permita sostener su esfuerzo y mantener la coherencia de los equipos.

Algunos éxitos ya se exportan. Estos primeros resultados traerán otros. Se ha producido un ahorro de horas de utilización de las máquinas, de horas de trabajo de los obreros participando de esta manera en la lucha permanente que las empresas francesas han de mantener junto con los poderes públicos para resolver el problema del empleo.

Resumiendo diremos que: Francia debe exportar para vivir. Sin materias primas, expuesta a la competencia de paises en fase de industrialización con salarios más bajos, no tiene otro recurso que su talento y sus técnicas para despuntar en la competición internacional.

Sucede que, debido a un juego de circunstancias históricas y sobre todo a los esfuerzos realizados con los supergeneradores, la energia nuclear francesa bien situada deberá explotarse al máximo.

Este artículo es una reproducción del publicado en la revista "Travaux», n. ${ }^{\circ} 517$.

\section{résumé} L'ENERGIE NUCLEAIRE ET L'INDUS-
TRIE EN FRANCE

A. Roux

Dans cet article, l'auteur fait une analyse des effets favorables que, pour l'industrie ont le developpement de l'énergie nucleaire et les interactions entre toutes les deux.

II fait allusion aux problemes de l'approvisionnement énergétique en France et au besoin de faire un effort en matière d'économie d'énergie, au développement de nouvelles sources et à la prioritè de l'établissement d'un important programme de centrales nucléaires

Ensuite, il analyse le rôle joué par l'industrie, par le Commissariat à l'Energie Ato mique et par l'Electricité de France, dans l'approvisionnement en matériaux nucléaires et dans leur transformation.

Finalement, l'auteur passe en revue les moyens humains et techniques, dont on dispose actuellement, et les investissements prevus pour un developpement national de cette énergie.

\section{summary}

NUCLEAR ENERGY AND INDUSTRY
IN FRANCE

IN FRANCE

In this article an analysis is made of the favorable effects for industry involved in the development of nuclear energy and the interaction between them.

Reference is made to the problem of energy supply in France and the need for an effort in energy savings, to the development of new sources and priority in the establishment of an important program of nuclear power plants.

Also, an analysis is made of the role of Industry, the Atomic Energy and Electricity Commission of France in the supply and processing of the nuclear materials.

Finally, a generat review of the manpower and technical means presently available is made, as well as an examination of the investments foreseen for local development investments
of this energy.

\section{zusammenfassung}

DIE KERNENERGIE UND DIE INDUSTRIE IN FRANKREICH A. Roux

In diesem Artikel werden die günstigen Wirkungen der Kernoder Atomenergie entwicklung auf die Industrie sowie die Beziehungen zwischen beiden untersucht.

Man erwähnt die Probleme Frankreichs bei der Energieversorgung und die Notwendigkeit einer Energieeinsparung, sowie die Entwicklung neuer Quellen und die Priorität bei der Einrichtung und Festlegung eines bedeutenden Programmes bezüglich der Kernkraftwerke.

Weiterhin werden die Aufgaben der Industrie, des Ausschusses für Atomenergie und Elektrizität in Frankreich und die Versorgung und Bearbeitung der nuklearen Materiale untersucht.

Abschliessend werden alle zur Zeit vorhandenen menschlichen und technischen Mittel sowie die vorgesehenen Investitionen zur nationalen Entwicklung dieser Energie zusammengefasst. 